\title{
ENERGY ESTIMATES AND THE WEYL CRITERION ON COMPACT HOMOGENEOUS MANIFOLDS
}

$$
\text { By }
$$

\section{S.B. Damelin}

\section{J. Levesley}

and

\section{Sun}

\section{IMA Preprint Series \# 2082}

(December 2005)

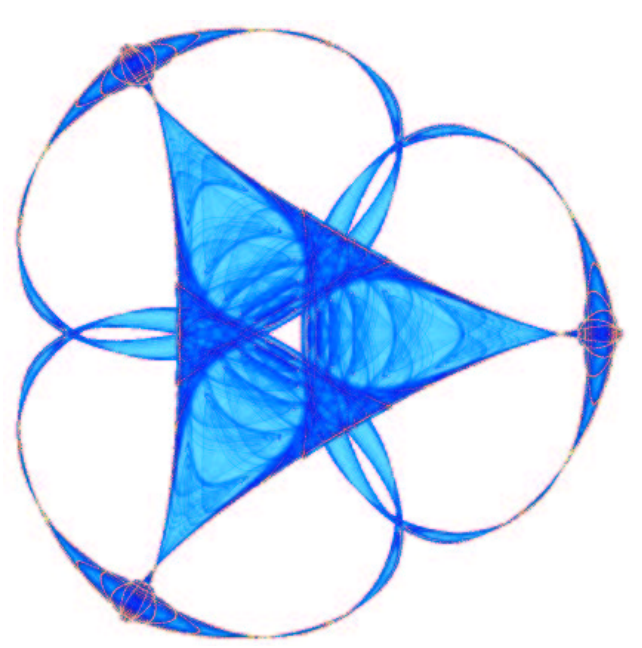

INSTITUTE FOR MATHEMATICS AND ITS APPLICATIONS

UNIVERSITY OF MINNESOTA 400 Lind Hall 207 Church Street S.E. Minneapolis, Minnesota 55455-0436

Phone: 612/624-6066 Fax: 612/626-7370

URL: http://www.ima.umn.edu 


\title{
Energy estimates and the Weyl criterion on compact homogeneous manifolds
}

\author{
S.B Damelin ${ }^{1}$, J. Levesley ${ }^{2}$ and X. Sun ${ }^{3}$ \\ 1 Institute for Mathematics and its Applications, University of Minnesota, 400 \\ Lind Hall, 207 Church Hill, Minneapolis, MN 55455, U.S.A. \\ damelin@ima.umn.edu \\ This author is supported, in part, by EP/C000285 and NSF-DMS-0439734. \\ 2 Department of Mathematics, University of Leicester, Leicester LE1 7RH, UK. \\ jl1@mcs.le.ac.uk \\ 3 Department of Mathematics, 10M Cheek Hall, Missouri State University \\ Springfield, MO 65897, U.S.A XSun@MissouriState.edu
}

\begin{abstract}
Summary. The purpose of this paper is to demonstrate how many results concerning approximation, integration, and density on the sphere can be generalised to a much wider range of manifolds $M$, namely the compact homogeneous manifolds. The essential ingredient is that invariant kernels (the generalisation of zonal or radial kernels) have a spectral decomposition in terms of projection kernels onto invariant polynomial subspaces. In particular, we establish a Weyl criterion for such manifolds $M$ and announce an energy result which generalizes work of Damelin and Grabner.
\end{abstract}

Keywords and Phrases: Compact Homogeneous Manifold, Energy, Harmonic, Invariant Kernels, Invariant Polynomial Subspaces, Numerical Integration, Projection Kernels, Uniform Distribution, Weyl

\section{Introduction}

Let $M$ be a $d \geq 1$ dimensional homogeneous space of a compact Lie group $G$ embedded in $\mathbb{R}^{d+r}$ for some $r \geq 0$. Then (see [4]), we may assume that $G \subset O(d+r)$, the orthogonal group on $\mathbb{R}^{d+r}$. Thus $M=\{g p: g \in G\}$ where $p \in M$ is a non-zero vector in $\mathbb{R}^{d+r}$. For technical reasons, we will assume that $M$ is reflexive. That is, for any given $x, y \in M$, there exists $g \in G$ such that $g x=y$ and $g y=x$.

Let $d(x, y)$ be the geodesic distance between $x, y, \in M$ induced by the embedding of $M$ in $\mathbb{R}^{d+r}$ (see [5] for details). On the spheres, this corresponds to the usual geodesic distance. A real valued function $\mathcal{K}(x, y)$ defined on $M \times$ $M$ is called a positive definite kernel on $M$, if for every finite subset $Y \subset M$, and arbitrary real numbers $c_{y}, y \in Y$, we have 


$$
\sum_{x \in Y} \sum_{y \in Y} c_{x} c_{y} \kappa(x, y) \geq 0 .
$$

If the above inequality becomes strict whenever the points $y$ are distinct, and not all the $c_{y}$ are zero, then the kernel $\kappa$ is called strictly positive definite. A kernel $\kappa$ is called $G$-invariant (or zonal) if $\kappa(g x, g y)=\kappa(x, y)$ for all $x, y \in M$ and $g \in G$. For example, if $M:=S^{d}$, the $d$ dimensional sphere realized as a subset of $\mathbb{R}^{d+1}$ and $G:=O(d+1)$, then all the $G$ invariant kernels have the form $\phi(x y)$, where $\phi:[-1,1] \rightarrow \mathbb{R}$, and where $x y$ denotes the usual inner product of $x$ and $y$ on $L_{2}(M)$. A kernel of the form $\phi(x y)$ is often called a zonal kernel on the sphere in the literature.

Let $\mu$ be a $G$-invariant measure on $M$ (which may be taken as an appropriately normalized 'surface' measure). Then, for real valued $f, g: L_{2}(M) \rightarrow \mathbb{R}$, we define an inner product with respect to $\mu$ :

$$
[f, g]=[f, g]_{\mu}:=\int_{M} f g d \mu
$$

and let $L_{2}(M)_{\mu}$ denote the space of all those functions from $L_{2}(M)$ into $\mathbb{R}$ for which the above inner product is finite. In the usual way, we identify 2 functions as being equal in $L_{2}(M)_{\mu}$, if they are equal almost everywhere $\mu$ wise.

Let $n \geq 0$ and $P_{n}$ be the space of polynomials in $d+r$ variables of degree $n$ restricted on $M$. Here, multiplication is taken pointwise on $\mathbb{R}^{d+r}$. The harmonic polynomials of degree $n$ on $M$ are $H_{n}:=P_{n} \bigcap P_{n-1}^{\perp}$. We may always (uniquely) decompose $H_{n}$ into irreducible $G$-invariant subspaces $H_{n, k}$, $k=1, \ldots, \nu_{n}$. Indeed, the uniqueness of the decomposition follows by the minimality of the $G$ invariant space, since a different decomposition would give subspaces contained in minimal ones leading to a contradiction.

Now, for $n \geq 0, k \geq 1$, let $Y_{n, k}^{1}, \ldots, Y_{n, k}^{d_{n, k}}$ be any orthonormal basis for $H_{n, k}$, and set

$$
Q_{n, k}(x, y):=\sum_{j=1}^{d_{n, k}} Y_{n, k}^{j}(x) Y_{n, k}^{j}(y) .
$$

Then $Q_{n, k}$ is the unique $G$-invariant kernel for the orthogonal projection $T_{n, k}$ of $L_{2}(M)_{\mu}$ onto $H_{n, k}$ acting as

$$
T_{n, k} f(x)=\int_{M} Q_{n, k}(x, y) f(y) d \mu(y) .
$$

For what follows, we require the following easily proved facts:

Lemma 1. Let $y, z$ be fixed points in $M$. Then

a.

$$
\int_{M} Q_{n, k}(y, x) Q_{n, k}(x, z) d \mu(x)=Q_{n, k}(y, z)
$$


b. $Q_{n, k}(x, x)=d_{n, k}$.

c. If $\kappa$ is a $G$-invariant kernel, then $\kappa(x, y)=\kappa(y, x)$.

Proof: Part (a) follows directly from the fact that $Q_{n, k}$ is the kernel for projection onto $H_{n, k}$.

Part (b) is a consequence of the equation

$$
Q_{n, k}(x, x):=\sum_{j=1}^{d_{n, k}} Y_{n, k}^{j}(x) Y_{n, k}^{j}(x) .
$$

Indeed, since $Q_{n, k}$ is $G$-invariant, it is $Q_{n, k}(x, x)$ constant on $M$. Integrating the last equation over $M$ and using the orthonormality of the $Y_{n, k}^{j}$, we then arrive at the required result.

The proof of Part (c) needs the reflexivity of $M$. Indeed, pick a $g \in G$ so that $g x=y$ and $g y=x$. Then

$$
\kappa(x, y)=\kappa(g y, g x)=\kappa(y, x)
$$

using the $G$-invariance of $\kappa$.

An important consequence of the above construction is that each irreducible subspace is generated by the translates of a fixed element. For this result on the sphere $S^{d}$, see, for instance, [1].

Proposition 1. Let $0 \neq Y \in H_{n, k}$. Then $H_{n, k}=\{Y(g \cdot) ; g \in G\}$.

Proof: It is clear that $V=\{Y(g \cdot) ; g \in G\}$ is a $G$-invariant subspace of $H_{n, k}$, and since $Y$ is not zero this is a non trivial subspace. But $H_{n, k}$ is irreducible, so that $V$ cannot be a proper subspace of $H_{n, k}$. Thus $V=H_{n, k}$.

Any $G$-invariant kernel $\kappa$, has an associated integral operator which we define by

$$
T_{\kappa} f(x)=\int_{M} \kappa(x, y) f(y) d \mu(y) .
$$

Then we have

Lemma 2. Let $\kappa_{1}$ and $\kappa_{2}$ be $G$-invariant. If $M$ is a reflexive space, $T_{\kappa_{1}} T_{\kappa_{2}}=$ $T_{\kappa_{1}} T_{\kappa_{2}}$.

Proof: Let $f \in L_{2}(M)_{\mu}$. Then

$$
\begin{aligned}
{\left[T_{\kappa_{1}} T_{\kappa_{2}} f\right](x) } & =\int_{M} \kappa_{1}(x, y)\left\{\int_{M} \kappa_{2}(y, z) f(z) d \mu(z)\right\} d \mu(y) \\
& =\int_{M} f(z)\left\{\int_{M} \kappa_{1}(x, y) \kappa_{2}(y, z) d \mu(y)\right\} d \mu(z) .
\end{aligned}
$$

Since the manifold is reflexive there is a $g \in G$ which interchanges $x$ and $z$. Thus, 


$$
\int_{M} \kappa_{1}(x, y) \kappa_{2}(y, z) d \mu(y)=\int_{M} \kappa_{1}(z, y) \kappa_{2}(y, x) d \mu(y),
$$

so that

$$
\begin{aligned}
{\left[T_{\kappa_{1}} T_{\kappa_{2}} f\right](x) } & =\int_{M} f(z)\left\{\int_{M} \kappa_{1}(z, y) \kappa_{2}(y, x) d \mu(y)\right\} d \mu(z) \\
& =\int_{M} \kappa_{2}(x, y)\left\{\int_{M} \kappa_{1}(y, z) f(z) d \mu(z)\right\} d \mu(y) \\
& =\left[T_{\kappa_{2}} T_{\kappa_{1}} f\right](x),
\end{aligned}
$$

where the penultimate step uses Lemma 1 (c). The changes of order of integration are easy to justify since the kernels are continuous and $f \in L_{2}(M)_{\mu}$.

We are now able to show that zonal kernels have a spectral decomposition in terms of projection kernels onto invariant polynomial subspaces. This is contained in

Theorem 1. If $M$ is a reflexive manifold, then any $G$-invariant kernel $\kappa$ has the spectral decomposition

$$
\kappa(x, y)=\sum_{n=0}^{\infty} \sum_{k=1}^{\nu_{n}} a_{n, k}(\kappa) Q_{n, k}(x, y)
$$

where

$$
a_{n, k}(\kappa)=\frac{1}{d_{n, k}} \int_{M} \kappa(x, y) Q_{n, k}(x, y) d \mu(y) .
$$

Here convergence is understood operatorwise in $L_{2}(M)_{\mu}$.

Proof: If $Y \in H_{n, k}$ then $T_{p_{n, k}} Y=Y$. Thus

$$
\begin{aligned}
T_{\kappa} Y & =T_{\kappa}\left(T_{Q_{n, k}} Y\right) \\
& =T_{Q_{n, k}}\left(T_{\kappa} Y\right) \in H_{n, k},
\end{aligned}
$$

since $T_{Q_{n, k}}$ is the orthogonal projection onto $H_{n, k}$. Here we have used Lemma 2.

Since $T_{\kappa}$ is a symmetric operator, it can be represented on the finite dimensional subspace by a symmetric matrix. Either this matrix is the zero matrix, in which case $a_{n, k}(\kappa)=0$, or $T_{\kappa}$ has non trivial range. Since the matrix is symmetric, it must have a non-zero real eigenvalue, $\gamma$ say, with associated eigenvector $Y$.

However, if $Y$ is an eigenvector then, so is $Y(g \cdot)$ for any $g \in G$ since

$$
\begin{aligned}
{\left[T_{\kappa} Y(g \cdot)\right](x) } & =\int_{M} \kappa(x, y) Y(g y) d \mu(y) \\
& =\int_{M} \kappa\left(x, g^{-1} y\right) Y(y) d \mu\left(g^{-1} y\right) \\
& =\int_{M} \kappa(g x, y) Y(y) d \mu(y)
\end{aligned}
$$


using the $G$-invariance of both $\kappa$ and $\mu$. But $Y$ is an eigenvector of $T_{\kappa}$, so that

$$
\left[T_{\kappa} Y(g \cdot)\right](x)=\gamma Y(g x)
$$

But, using Proposition 1 we see that $H_{n, k}$ is an eigenspace for $T_{\kappa}$ with single eigenvalue $\gamma$. We can compute $\gamma$ by evaluating $T_{\kappa}$ on $Q_{n, k}(\cdot, y)$ for fixed $y$ :

$$
\int_{M} \kappa(z, x) Q_{n, k}(x, y) d \mu(x)=\gamma Q_{n, k}(z, y) .
$$

Setting $z=y$ and using Lemma 1 (b) we have

$$
\gamma=\frac{1}{d_{n, k}} \int_{M} \kappa(y, x) Q_{n, k}(x, y) d \mu(x),
$$

and the appropriate form for $\gamma$ follows using the symmetry of $G$-invariant kernels (Lemma 1 (c)).

Henceforth, we assume that $a_{n, k}(\kappa) \geq 0$ for all $n, k$, and

$$
\sum_{n=0}^{\infty} \sum_{k=1}^{\nu_{n}} d_{n, k} a_{n, k}(\kappa)<\infty
$$

Thus $\kappa$ is bounded on $M \times M$, is positive definite there and hence absolutely integrable on $M$, ie, there exists finte $C$ such that uniformly for all $x \in M$,

$$
\int_{M}|\kappa(x, y)| d \mu(y) \leq C
$$

In what follows, such kernels will be called admissible. The archetype for admissible kernels is the Riesz kernel

$$
\kappa(x, y)=\|x-y\|^{-s}, \quad s>0, \quad x, y \in M,
$$

where $\|\cdot\|$ is the Euclidean norm in $\mathbb{R}^{d+r}$.

We will also need to assume the following simple consequence of Theorem 4. See [3] for the proof.

Lemma 3. (Funk-Hecke formula) For any $x, y \in M$,

$$
\int_{M} Q_{n, k}(x, y) Q_{n, k}(y, z) d \mu(y)=Q_{n, k}(x, z) .
$$

\section{The Weyl criterion}

In this section, we will prove the equivalence of two definitions of uniform distribution of points. Following is our main result of this section. 
Theorem 2. The following two definitions for a uniformly distributed sequence are equivalent.

a. A sequence $\left\{x_{l}: l \in \mathbf{N}\right\}$ is uniformly distributed on $M$ if and only if

$$
\lim _{N \rightarrow \infty} \frac{1}{N} \sum_{l=1}^{N} Y_{n, k}^{j}\left(x_{l}\right)=0
$$

for all $n \geq 0$ and $1 \leq k \leq \nu_{n}, 1 \leq j \leq d_{n, k}$.

b. Let $\kappa$ be a positive definite zonal kernel on $M$. A sequence $\left\{x_{l}: l \in \mathbf{N}\right\}$ is uniformly distributed on $M$ if and only if

$$
\lim _{N \rightarrow \infty} \frac{1}{N} \sum_{l=1}^{N} \kappa\left(x_{l}, y\right)=a_{0,0}(\kappa),
$$

holds true uniformly for $y \in M$.

Proof: Using the series expansion for $\kappa$ we have

$$
\frac{1}{N} \sum_{l=1}^{N} \kappa\left(x_{l}, y\right)=\sum_{n=0}^{\infty} \sum_{k=1}^{\nu_{n}} a_{n, k}(\kappa) \sum_{j=1}^{d_{n, k}} Y_{n, k}^{j}(y)\left(\frac{1}{N} \sum_{l=1}^{N} Y_{n, k}^{j}\left(x_{l}\right)\right) .
$$

Suppose $\left\{x_{l}: l \in \mathbf{N}\right\}$ is uniformly distributed by (a). Now, the right hand side of the last equation is majorised by

$$
\sum_{n=0}^{\infty} \sum_{k=1}^{\nu_{n}} a_{n, k}(\kappa) \frac{1}{N} \sum_{l=1}^{N}\left|Q_{n, k}\left(x_{l}, y\right)\right| \leq \sum_{n=0}^{\infty} \sum_{k=1}^{\nu_{n}} d_{n, k} a_{n, k}(\kappa),
$$

using Lemma 1 (a). This is bounded from (1).

Therefore, using the dominated convergence theorem we can pass the limit over $N$ through the sum to give

$$
\begin{aligned}
& \lim _{N \rightarrow \infty} \sum_{n=1}^{\infty} \sum_{k=1}^{\nu_{n}} a_{n, k}(\kappa) \sum_{j=1}^{d_{n, k}} Y_{n, k}^{j}(y)\left(\frac{1}{N} \sum_{l=1}^{N} Y_{n, k}^{j}\left(x_{l}\right)\right) \\
& =\sum_{n=1}^{\infty} \sum_{k=1}^{\nu_{n}} a_{n, k}(\kappa) \sum_{j=1}^{d_{n, k}} Y_{n, k}^{j}(y) \lim _{N \rightarrow \infty}\left(\frac{1}{N} \sum_{l=1}^{N} Y_{n, k}^{j}\left(x_{l}\right)\right) \\
& =0,
\end{aligned}
$$

by assumption. Thus

$$
\frac{1}{N} \sum_{l=1}^{m} \kappa\left(x_{l}, y\right)=a_{0,0}(\kappa)
$$

uniformly in $y$, so that $\left\{x_{l}: l \in \mathbf{N}\right\}$ is uniformly distributed by (b). 
Conversely suppose that $\left\{x_{l}: l \in \mathbf{N}\right\}$ is uniformly distributed by (b). Then, as in (2), we have

$$
\frac{1}{N^{2}} \sum_{m=1}^{N} \sum_{l=1}^{N} \kappa\left(x_{m}, x_{l}\right)=\sum_{n=0}^{\infty} \sum_{k=1}^{\nu_{n}} a_{n, k}(\kappa) \sum_{j=1}^{d_{n, k}}\left(\frac{1}{N} \sum_{l=1}^{N} Y_{n, k}^{j}\left(x_{l}\right)\right)^{2} .
$$

Now, for each $x_{m}$, by hypothesis

$$
\lim _{N \rightarrow \infty} \frac{1}{N} \sum_{l=1}^{N} \phi\left(x_{m}, x_{l}\right) \rightarrow \int_{M} \phi\left(x_{m}, x\right) d \mu(x)=a_{0,0}(\kappa) .
$$

Thus,

$$
\lim _{N \rightarrow \infty} \frac{1}{N^{2}} \sum_{m=1}^{N} \sum_{l=1}^{N} \phi\left(x_{l}, x_{j}\right) \rightarrow \int_{M} \phi\left(x, x_{j}\right) d \mu(x)=a_{0,0}(\kappa) .
$$

Therefore

$$
\lim _{N \rightarrow \infty} \sum_{n=1}^{\infty} \sum_{k=1}^{\nu_{n}} a_{n, k}(\kappa) \sum_{j=1}^{d_{n, k}}\left(\frac{1}{N} \sum_{l=1}^{N} Y_{n, k}^{j}\left(x_{l}\right)\right)^{2}=0,
$$

and since $a_{n, k}(\kappa)>0, n \in \mathbf{N}$ and $1 \leq k \leq \nu_{n}$, it must be that

$$
\lim _{N \rightarrow \infty} \frac{1}{N} \sum_{l=1}^{N} Y_{n, k}^{j}\left(x_{l}\right)=0,
$$

so that $\left\{x_{l}: l \in \mathbf{N}\right\}$ is uniformly distributed by (a).

\section{Energy on manifolds}

In this last section, we are interested in studying errors of numerical integration of continuous functions $f: M \rightarrow \mathbb{R}$ over a countable point set $Z \subset M$ of cardinality $N \geq 1$. In particular, we seek a generalization of results of Damelin and Grabner in [2]. More precisely, given an admissible kernel $\kappa$ and such a point set $Z$, we define the discrete energy

$$
E_{\kappa}(Z)=\frac{1}{N^{2}} \sum_{\substack{y, z \in Z \\ y \neq z}} \kappa(y, z)
$$

and for the normalized $G$ invariant measure $\mu$ on $M$, denote by

$$
R(f, Z, \mu):=\left|\int_{M} f d \mu-\frac{1}{N} \sum_{y \in Z} f(y)\right|
$$


the error of numerical integration of $f$ with respect to $\mu$ over $M$.

For an admissible kernel $\kappa$ and probability measure $\nu$ on $M$, we define the energy integral

$$
\mathcal{E}_{\kappa}(\nu)=\int_{M} \int_{M} \kappa(x, y) d \nu(x) d \nu(y) .
$$

We have

Lemma 4. The energy integral $\mathcal{E}_{\kappa}(\nu)$ is uniquely minimised by the normalized $G$ invariant measure $\mu$.

Proof: Since $\kappa$ is positive definite, $\mathcal{E}_{\kappa}(\nu) \geq 0$ for every Borel probability measure $\nu$. Also, a simple computation shows that $\mathcal{E}_{\kappa}(\mu)=a_{0,0}(\kappa)$.

Next, we observe that we have using Lemma 3 , for all probability measures $\sigma$ on $M$,

$$
\begin{aligned}
\mathcal{E}_{\kappa}(\sigma) & =\int_{M} \int_{M}\left\{\sum_{n=0}^{\infty} \sum_{k=1}^{\nu_{n}} a_{n, k}(\kappa) P_{n, k}(x, z)\right\} d \sigma(x) d \sigma(z) \\
& =a_{0,0}(\kappa)+\sum_{n=1}^{\infty} \sum_{k=1}^{\nu_{n}} a_{n, k}(\kappa) \int_{M} \int_{M} P_{n, k}(x, z) d \sigma(x) d \sigma(z) \\
& =a_{0,0}(\kappa)+\sum_{n=1}^{\infty} \sum_{k=1}^{\nu_{n}} a_{n, k}(\kappa) \int_{M} \int_{M} \int_{M} P_{n, k}(x, y) P_{n, k}(y, z) d \mu(y) d \sigma(x) d \sigma(z) \\
& =a_{0,0}(\kappa)+\sum_{j=1}^{\infty} \sum_{k=1}^{\nu_{n}} a_{n, k}(\kappa) \int_{M}\left\{\int_{M} P_{n, k}(x, y) d \sigma(x)\right\}^{2} d \mu(y)
\end{aligned}
$$

using the symmetry of the kernel $P_{n, k}(x, y)$.

Thus, for $\nu$ to minimise $\mathcal{E}_{\kappa}$,

$$
\int_{M} P_{n, k}(x, y) d \nu(x)=0, \quad k=1, \ldots, \nu_{n}, \quad n=1, \ldots
$$

Hence, since $\mu$ also annihilates all polynomials of degree $\geq 0, \nu-\mu$ annihilates all polynomials. Because the polynomials are dense in the continuous functions, we see that $\nu-\mu$ is zero and the result is proved.

Heuristicly, one expects that a point distribution $Z$ of minimal energy gives a discrete approximation to the measure $\mu$, in the sense that the integral with respect to the measure is approximated by a discrete sum over the points of $Z$. For the sphere, this was shown by Damelin and Grabner in [2] for Riesz kernels. The essence of our main result below is that we are able to formulate a general analogous result which works on $M$ and for a subclass of admissible kernels $\kappa$. To describe this result, we need some more notation.

Let $\sigma_{\alpha}$ be a sequence of kernels converging to the $\delta$ distribution (the distribution for which all Fourier coefficients are unity) as $\alpha \rightarrow 0$. Let $\kappa$ be admissible and for $\alpha<\alpha_{0}$ for some fixed $\alpha_{0}$, we wish the convolution $\kappa_{\alpha}=\kappa * \sigma_{\alpha}$ to have the following properties: 
a. $\kappa_{\alpha}$ is positive definite

b. $\kappa_{\alpha}(x, y) \leq \kappa(x, y)$ for all $x, y \in M$.

If the above construction is possible, we say that $\kappa$ is strongly admissible. Besides Riesz kernels on $d$ dimensional spheres see [2,3], we have as a futher natural example on the 2 -torus embedded in $\mathbb{R}^{4}$, strongly admissible kernels defined as products of univariate kernels:

$$
\kappa(x, y)=\rho\left(x_{1}, y_{1}\right) \rho\left(x_{2}, y_{2}\right), \quad x_{1}, y_{1}, x_{2}, y_{2} \in S^{1},
$$

where

$$
\rho(s, t)=|1-s t|^{-1 / 2}, s, t \in(-1,1) .
$$

See [3] for further details. We are now able to announce our main result of this section. See [3] for the proof and further results.

Theorem 3. Let $\kappa$ be strongly admissible on $M$ and $Z \subset M$ be a point subset of cardinality $N \geq 1$. Fix $x \in M$. If $q$ is a polynomial of degree at most $n \geq 0$ on $M$ then, for $\alpha<\alpha_{0}$,

$|R(f, Z, \mu)| \leq \max _{j \leq n, l \leq h_{j}} \frac{1}{\left(a_{j, l}\left(\kappa_{\alpha}\right)\right)^{1 / 2}}\|q\|_{2}\left(E_{\kappa}(Z)+\frac{1}{N} \kappa_{\alpha}(x, x)-a_{0,0}\left(\kappa_{\alpha}\right)\right)^{1 / 2}$.

\section{References}

1. Personal Communication.

2. S. B. Damelin and P. Grabner, Numerical integration, energy and asymptotic equidistributon on the sphere, Journal of Complexity, 19(2003), 231-246.

3. S. B. Damelin, J. Levesley and X. Sun, Quadature estimates for compact homogeneous manifolds, manuscript.

4. G. D Mostow, Equivariant embeddings in Euclidean space, Ann. Math, 65(1957), 432-446.

5. J. Levesley and D. L. Ragozin, The density of translates of zonal kernels on compact homogeneous spaces, J. Approx Theory, 103(2000), 252-268. 